\title{
Measurement of Railway Track Geometry: A State-of-the-Art Review
}

\author{
András Farkas ${ }^{1 *}$ \\ ${ }^{1}$ Institute of Entrepreneurship Development, Óbuda University, 1084 Budapest, Tavaszmező u. 15-17, Hungary \\ * Corresponding author, e-mail: farkas.andras@kgk.uni-obuda.hu
}

Received: 04 April 2019, Accepted: 13 May 2019, Published online: 22 October 2019

\begin{abstract}
The worldwide increase in frequency of traffic for passenger trains and the rise of freight trains over the recent years necessitate the more intense deployment of track monitoring and rail inspection procedures. The wheel-rail contact forces, induced by the static axle loads of the vehicle and the dynamic effects of ground-borne vibration coming from the track superstructure, have been a significant factor contributing to the degradation of the railway track system. Measurements of track irregularities have been applied since the early days of railway engineering to reveal the current condition and quality of railway lines. Track geometry is a term used to collectively refer to the measurable parameters including the faults of railway tracks and rails. This paper is aiming to review the characteristics of compact inertial measurement systems (IMUs), their components, installation, the basic measures of the quality of the track using motion sensors, like accelerometers, gyroscopes and other sensing devices mounted on different places of the vehicle. Additionally, the paper briefly discusses the fundamentals of inertial navigation, the kinematics of the translational and rotational train motions to obtain orientation, velocity and position information.
\end{abstract}

Keywords

inertial measurement sensors, train motion kinematics, railway track geometry condition monitoring

\section{Introduction}

In recent years, considerable research has been done in many countries related to condition monitoring of railway track geometry and investigating the performance of riding quality. Among others, in the paper of Weston et al. (2015), authors presented an excellent overview about the current developments of such systems installed on board of railway coaches running in regular operation all over the country in Great Britain. A detailed reasearch was reported from Japan about the use of a vertical displacement measuring system mounted on the bogie and supplemented by an additional sensor from the bogie to the axlebox to monitor rail geometry (Yazawa and Takeshita, 2002). Jochim and Lademann (2009) in Germany, gave an overall description about the track geometry characteristics. Ackroyd et al. (2002) reported high accelerations measured both on the bogie and the body using a condition sensing system instrumented on Acela trains in the USA. A stochastic simulation was done in Switzerland, on the Gotthard line, to investigate the wear propagation process for both the rails and the wheels (Szabó and Zobory, 1998).
Here, the authors gave estimates of the magnitude and its distribution of the right/left rails' wear and wheels' wear along the track for straight, curved and transition sections. Zobory and Péter (1987) proposed a time- and state dependent, seven degree-of-freedom model with its corresponding system of motion equations including several non-linearities, and examined the behavior of a braking vehicle on the dynamic effects of track unevennesses by assuming stationary, stochastic track records. A comprehensive overview about the whole topic can be found in the research report of Nielsen et al. (2013).

The objective of this paper is twofold. One goal is to discuss the operative aspects of measurement technologies of railway track geometry by giving an up-to-date overview based on a state-of-the-art literature review. Another goal is to point and stimulate towards directions for further research in this area having utmost importance for practitioners as well. The organization of the survey is as follows. The main characteristics of the track measurement systems will be presented in Section 2. 
In Subsection 2.1, the track geometry faults are summarized; in Subsection 2.2, the measuring systems and their elements will be discussed with special focus on the Inertial Measurement Units (IMUs); in Subsection 2.3, some types of these devices are described; in Subsection 2.4, the placement of these intruments on the measuring vehicle will be analyzed; in Subsection 2.5, the unavoidable errors emerging in the course of signal processing are discussed; in Subsection 2.6, the use of the two basic procedures of track measurement, the continuous in-service vehicles and the dedicated measurement coaches, are compared; in Subsection 2.7, the characteristics of accelerometers and gyroscopes will be given. In Section 3, the kinematics of the inertial navigation systems are briefly summarized; and, in Section 4, the train motion depending on the track geometric (and geographic) position will be described.

\section{Characteristics of railway track measurements}

This section provides the reader a concise overview of the characteristics of railway track measurement systems.

\subsection{Track geometry faults}

Railways require to maintain the highest ride comfort and safety standards for the status of the trains/carriages and facilitate maintenance planning of the tracks what is called on-board condition monitoring and control. Integrated track control and inspection encompass a variety of parameters, e.g. longitudinal level, track gauge, cant, alignment, twist - related to the track geometry; then corrugation, squat, wheel slipping points, dipped joints/welds, cyclic top derailments - grouped into the set of the short rail surface defects; and, measurements of dynamics and driving comfort parameters. As a rule of thumb, if the geometry of the track is known for each $0.2 \mathrm{~m}$ section and a fault can be localized within $3 \mathrm{~m}$ in an absolute position, then such a monitoring system is well suited to the needs of railway companies in order to preserve the condition of tracks.

After the initial construction of the track, the geometry of the track begins to deviate from its original, called design geometry. This process is known as track degradation. Degradation can occur for a number of reasons (Yeo, 2017): Firstly, the vertical geometry of the track tends to degrade over time (which was originally almost flat along a $\sim 70 \mathrm{~m}$ length of track). Secondly, the track stiffness, which can strongly affect the track's vertical profile, and its extreme values can lead to track failures. Deflection typically varies from a fraction of a millimeter to tens of millimeters in extreme cases. The static axle load of the train is the load on the wheel pairs as when the train is stationary, whereas the dynamic axle load of the train increases as the speed of the train set increases.

Geometric track defects are termed track irregularities (usually measured in displacement) which originates in the contact force caused by a continuous interaction between the wheel and the rail and the sleeper's deterioration. This is the source of the dynamic excitation which causes oscillations and vibrations for both the vehicle and the track. Track irregularities are usually grouped into two categories: $\lambda>1$ meter's are said the long (including mid) wavelength irregularities and $\lambda<1$ meter's are said the short wavelength irregularities. By Xing et al. (2015), long wavelength track irregularities affect railway operation strongly and impact vehicle stability and comfort. They are emerging from roadbed deformations due to soil compaction of multiple passages of heavy train sets, but they produce no shocks. The vehicle responses are typically low-frequency oscillations (ZG Optique, 2016).

When the train passes through short track defects having poor finishing quality, axle-box accelerations may produce $100 \mathrm{~g}$ or even more (Molodova et al., 2011). However, earlier investigations done by Weston et al. (2007) found that the long wavelength irregularities produce much less than $1 \mathrm{~g}$ axlebox accelerations. Therefore, they claimed to install sensors on axleboxes which have a low limit on lower bandwidth. To get usable long wavelength outputs they should have high linearity, and very low noise as well.

\subsection{Measuring devices of track irregularities}

The typical form of an Inertial Measurement Unit (IMU) contains three accelerometers and three gyroscopes and additionally, depending on the heading requirement, three magnetometers. An IMU should be mounted into a three dimensional orthogonal system. Such a configuration (a full six degree-of-freedom IMU), for a vehicle moving along the track, makes it possible to provide 3-D position and velocity information by measuring linear acceleration and rotation (gimbal rates) for the vehicle in the directions of each axis. Through the time of the measurements, there is a high sampling rate, usually at frequencies that are even higher than $100 \mathrm{~Hz}$. The accelerometers are mounted with their sensitive axes perpendicular to one another, i.e. mutually perpendicular. Gyros have specific objectives. A gyroscope serves as a proper tool for providing accurate information on the attitude and the heading of the body and/or the bogie with respect to a predetermined reference frame (see it in Section 3). Utilizing information that are 
related to linear accelerations and rotation rates, velocity, and hence position of the vehicle can also be derived.

Nowadays MEMS, Micro-Electro-Mechanical-Systems have become widely available due to their small sizes and low costs. They contain inertial measurement sensors (3-D accelerometers and 3-D gyroscopes) and have a very high sensitivity. They resist undesired vibration and shock, but in some cases, they suffer from limited accuracy. MEMS sensors can be placed into well sheltered and low weight boxes and then they are assembled onto the axlebox, or the bogie, or the body of the vehicle.

Such MEMS inertial sensing devices are most often used in compact Strapdown Inertial Navigation Systems (SINS) equipped with a receiver used for accommodating remote sensing signals emitted by satellite navigation systems. A satellite navigation system also has MEMS accelerometers and fiber-optic gyros. A SINS is usually mounted on the vehicle frame, or eventually, on the bottom of the carriage. This complex system supplies fundamental information for the navigation of the vehicle, i.e. position, velocity, attitude (heading, bank angle, slope, rotation rates) and relative displacements of the vector of acceleration and the angular rates with respect to the SINS frame (ZG Optique, 2016). SINS calculates the railroad track momentum gradient, the superelevation and crosslevel (positive and negative cant) and expressed them by numerical terms and optical system data (ZG Optique, 2016).

For example, a contactless optical system, developed by the Swiss ZG Optique SA for determining the attitude, consists of three radiating sources and three receivers (ZG Optique, 2016). They are installed on the bottom of the railway carriage body and on the bogie frame. This manner, an optical system without a direct contact is established to measure mutual orientation of the vehicle ( $\mathrm{ZG}$ Optique, 2016). A satellite receiver is also assembled on the top of the car body and IMUs are mounted on the bottom of the body and on the carriage frame (ZG Optique, 2016). Nevertheless, to set up such an optical configuration, which, as its usual setting, includes a laser scanner to monitor and determine the profiles of each rail, is expensive, moreover, it is very difficult to keep this optical sensor clear in the dirty operational environment (Xing et al., 2015). Yet this contactless technique is popular for railways, because it is effective and quick and is capable of performing long rail defects with lessened wear.

As a direct monitoring of the track defects a video system can be used with digitally sensing video cameras placed on the body frame and located inside the wheel pairs.
Periodic track faults significantly affect the dynamic loads coming from the primary suspension of the vehicle and caused by the wheel-rail interaction. This is true for both spring-borne and non-spring-borne loading. Such optical systems can provide very accurate measurements (to within $0.1 \mathrm{~mm}$ ) of the track geometry, but could be much more expensive to maintain.

An odometer is a tool to measure the distance taken by the vehicle. This device is installed on one of the wheel pairs. Typically a tachometer measures the rotation speed of a non-driven axle belonging to a wheel set usually in revolution/minute. British railway engineers, Weston et al. (2015:p.1064), have asserted that "The speed of rotation of an unpowered wheel and the odometer is the integration of the tachometer with respect to time multiplied by the wheel circumference to convert rotations per unit time into distance.". Distance measurement of a traveling vehicle is done in a direct way applying distance sensors. It should be noted that it is hard to measure the movements, say, only into the vertical direction, since the ends of the sensor will surely have certain lateral and longitudinal displacements as well. In this respect, Weston et al. (2015:p.1066) reported that "The capacitative or the inductive displacement sensors are more robust but can only measure small displacements.".

A complete process control system (PCS) is comprised of electronic, optical and computerized units. It is displayed in Fig. 1 (where their typical components are described in the caption).

In recent decades, railways have begun to install an antenna on the roof of the vehicle as a key element of

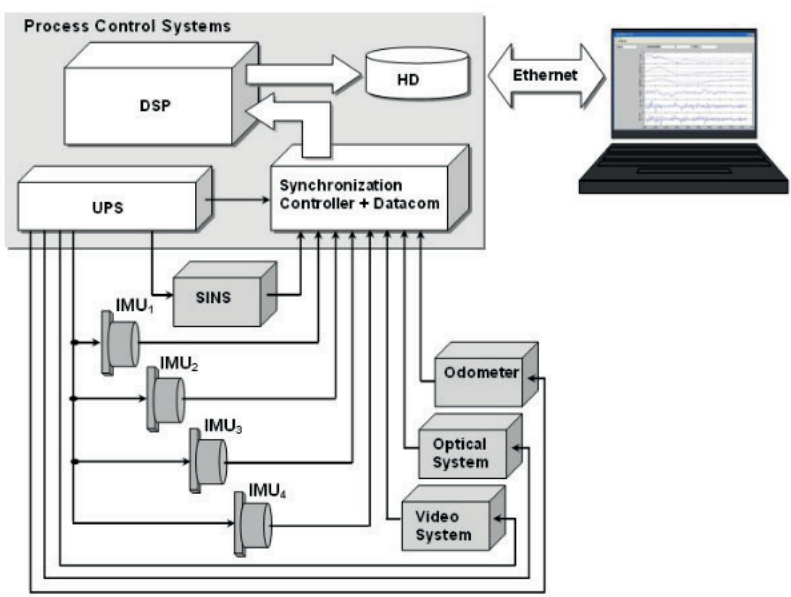

Fig. 1 "A process control system (PCS) comprises an uninterruptible power supply (UPS), signal synchronization and transmission board, digital signal processor (DSP), and hard disk (HD) packaged in a metal casing, and has USB, Ethernet and other interfaces." (ZG Optique, 2016:p.2) 
their Global Navigation Satellite System (GNSS) in order to get more accurate information to determine position. Indeed, measurements have proven that by combining a tachometer outcome with GPS/GNSS emitted data offers a remarkable positive change in exploring unbiased position of track condition data. To obtain absolute position information, even using a Kalman filtering technique, however, remains henceforward a crucial data acquisition task for railways (Mirabadi et al., 2003).

\subsection{Types of measuring devices}

As we discussed in the previous section, an IMU is a frequently used measurement tool of railway companies which consists of a triaxial accelerometer and a triaxial gyroscope. In accelerometers, usually a damped silicon mass is suspended by a spring where the inflowing kinetic energy is converted to an electrical signal by either a piezoelectric, or a piezoresistive, or a capacitive part. Accelerometers of piezoelectric mode are composed of ceramics or are being made of single crystals, while accelerometers having a capacitive form utilize mainly a micro-machined sensing element made of silicon. Latter configuration far surpasses the performance of the other accelerometers within the range of low frequencies.

A gyroscope is a rotation sensing device which stands for helping to specify orientation based on Earth's gravity. One typical type of gyroscope is made by suspending a relatively massive rotor assembled onto a spinning axis in the center of a larger and more stable wheel inside three rings called gimbals. Gyros have especially high stability to balancing themselves when the vehicle speed is very high. An other beneficial property of gyroscopes is that the direction of the high speed rotation axis of their central rotor remains invariant at high speeds.

The structure of MEMS sensors is extremely simple. They consist of a cantilever beam supplemented by a proof mass. It follows from this fact that any effort has been made towards reducing their purchasing costs has implied less accuracy in the performance of an IMU as a whole. Still, the MEMS's position and orientation estimates are accurate on a short time scale, but suffer from errors (drift, random walk) over longer time scales (Mirabadi et al., 2003). These fundamental facts related to the limited capabilities of MEMS sensors are often forgotten by railway staffs who are responsible for track monitoring and inspection.

Some railways employ optical measurement systems, using either laser scanners or a combination of lasers and video cameras. Basically these systems are very accurate in measuring track geometry, but too expensive to maintain them in an in-service railway operation because they require frequent cleaning.

It should also be remarked that even if the orientation estimate has insignificant errors only, yet it may experience extraordinarily large bias in the acceleration values. This fact will magnify errors in the velocity and position estimation process resulting very poor outputs. From an engineering point of view, in the lack of use of more effective rate gyros (FOG or Ring-Laser) or not to adding an ouside satellite based navigation system, to achieve accurate dead-reckoning is generally impossible. As shown by a profound experimental research at CH Robotics (2012) "An angle error of even one degree will cause the estimated velocity to be off by $1.7 \mathrm{~m} / \mathrm{s}$ after 10 seconds, and the position to be off by 17.1 meters in the same amount of time. After a single minute, one degree of angle error will cause the position estimate to be off by almost a full kilometer. After ten minutes, the position will be off by 62 kilometers." (CH Robotics, 2012:p.6).

\subsection{Placement of measuring devices on the vehicle}

The choice for the right places on the vehicle and the appropriate type of the measuring devices are a matter of utmost importance. To find a satisfactory solution to these problems is dependent on what kind of on-board condition monitoring and riding quality control system are desired to implement taking into consideration the type of track faults intending to be detected as well as the operational issues of maintanability. Yazawa and Takeshita (2002) reported that accelerometers mounted on the axlebox are regularly used on Japanese railway lines on high-speed trains. However, such an installation of acceleration sensors appears to be infavorable due to the difficulties of their maintenance tasks. Weston et al. (2015) described their experiences with sensors installed on axleboxes. For example, under very harsh weather conditions, they can be frozen, or exposed to a windstorm with a force over 100 $\mathrm{mph}$, or may warm up too strongly from the bearing.

Many authors in this field of interest believe axlebox accelerometers are the appropriate manner in helping to describe the vertical rail profile of track geometry correctly. Yet, even if we gain a perfect identification of the profiles of the rails, a double integration to derive displacement is required, which induces some inherent difficulties that will be discussed in Subsection 2.5. A slightly more robust solution is to lay the inertial sensors on the bogie frame and put optical sensors looking at the rails 
(Weston et al., 2015). Laterally mounted axle-box accelerometers can be used to measure short wavelength lateral defects such as poor rail alignment at switches and crossings (Ward et al., 2011).

Roberts et al. (2004) have written how they started to experiment with the placement of sensors onto different locations on the vehicle, in the mid-2000s. They raised a seemingly strange question as to whether the explicit exploration of geometry the track is explicitly needed at all. In this context, Roberts et al. (2004) have asserted that "If the bogie follows a smooth trajectory down the track, then the track geometry must be reasonably good. Conversely, poor track geometry almost always leads to something detectable in the trajectory of the bogie.". Some experiments have been carried out to investigate the motion of the bogie to ignore the primary suspension (Weston et al., 2007).

Mounting a single IMU directly onto the bogie frame results in a much simpler installation process. A bogiemounted IMU gives acceptable measurement results, particularly when the exact geometry does not need to be reconstructed (Weston et al., 2007). It is suitable for measurement of mid-wavelength (1-3 m) geometry features. Instrumentation mounted in this way is better suited to in-service installation, as it is less obtrusive and easier to remove and re-attach when the bogie requires maintenance.

In the sequel, we will take over several findings have been reported in the outstanding work of Yeo (2017). Using a bogie-mounted IMU, the very short wavelength geometry features are filtered out by the primary suspension. Some short wavelength defects are filtered out by the dynamic effects of the bogie, which could result in some loss of fidelity. The point of measurement is before the secondary airbag suspension between the bogie and the coach of the train, allowing much shorter wavelength measurements to be recorded than if the IMU were mounted on board the coach. The ride quality, however, is best measured from on board the car itself, as this is where passengers would be sitting or standing (Yeo, 2017).

The IMU itself is usually mounted on top of the bogie, using four tapped holes provided for the addition of extra equipment to the bogie (Yeo, 2017). The positioning of the IMU on the bogie is displayed in Fig. 2.

In order to reconstruct the geometry of the track, a better approach when using inertial sensors, seems to be to install the instrumentation on the bogie frame. It may come up also the top of the axlebox of the vehicle. These placements move the measurement point to below the secondary suspension. Often the primary suspension is very stiff, meaning that measurements taken on the bogie are close to those experienced at axlebox level. Instrumentation of the axlebox appears to be the best way of detecting very short wavelength features such as corrugation and squats. Bogie instrumentation is appropriate for providing measurements of mid-wavelength geometry features, as the primary suspension and the bogie itself provide a natural lowpass filtering of the accelerations experienced (Yeo, 2017).

If linear acceleration is used to measure vertical rail profile, then, a sensor on the bogie over the axlebox should be placed, and the vertically sensed displacement to the axlebox from the accelerometer. This combination is an example of censor fusion. King (2004) argued that "Moving the accelerometer from the axlebox to the bogie isolates the accelerometer from the worst impulsive accelerations, and reduces the range with which the accelerometer has to contend. However, the displacement transducer is then vulnerable." (King, 2004:p.18).

Sensor combination contributes to the improvement of measurement accuracy considerably. As an other example for censor fusion, Trehag et al. (2010) reported that GNSS data combined with yaw rate sensor and tachometer data to measure curvature produces a completely bias-free output, where curvature is computed as the ratio between the yaw rate and the speed of the vehicle. Nevertheless, the speed of the train impacts the result, since, at low speed, the yaw rate gyro is very sensitive to drifting offset phenomenon. which affects the curvature estimate. A curvature information for the long-run can be obtained from the GNSS data that may modify the offset error at low speeds provided that a correct mathematical model was setting up by the analyst. These investigations require to apply both linear and nonlinear filtering (Trehag et al., 2010).

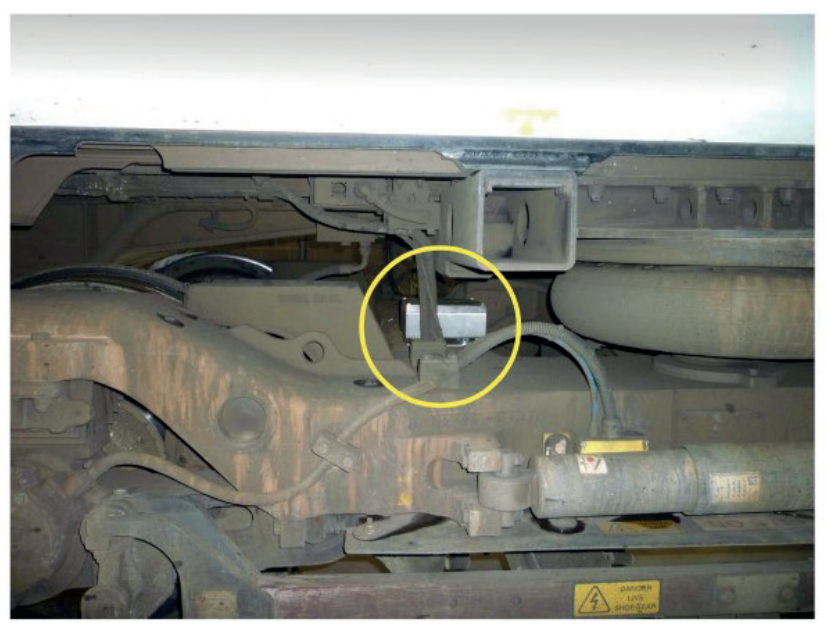

Fig. 2 IMU positioning on the bogie (Yeo, 2017:p.48) 
Similarly to track geometry monitoring, the integration of axlebox, bogie- and body- mounted measuring devices can help to reveal the current state of the structural components of the bogie and to identify the absolute speed of the moving train (Charles et al., 2008).

In the course of a series of experiments Weston et al. (2007) applied a bogie-mounted pitch rate gyroscope to derive the average vertical alignment and axlebox mounted accelerometers to compute the defects wavelength. This setting made it possible to analyze from $5 \mathrm{~m}$ to $70 \mathrm{~m}$ long wavelengths with high precision. In general, if we wish to monitor short wavelengths, then axlebox accelerometers should be used. Due to the fact that a long wavelength irregularity produces less than $1 \mathrm{~g}$ accelerations the axlebox accelerator sensor must have a low limit on lower bandwidth, high linearity and emitting extremely low noise effects in order to obtain reliable long wavelength output.

According to Xing et al. (2015) "Assuming that the leading and trailing wheel-sets follow the vertical track geometry and the primary suspension is infinitely stiff, the bogie pitch is determined by the vertical positions of the leading and trailing wheel-sets divided by the bogie wheelbase." (Xing et al., 2015:p.219). Based on some geometric considerations (e.g. spatial filtering) they stated that the bogie pitch cannot sense wavelength irregularities whose lengths are just equal to the bogie wheelbase (Xing et al., 2015).

Originally in Destek (1974), and later in Zobory and Zábori (1996), then in Zobory (2015), the authors expressed their belief that to look upon railway tracks as being described by pure geometry alone would be an outdated and professionally inappropriate view. Such an approach ignores the system dynamics aspects, i.e. the railway induced ground-borne vibrations result from the interaction between the moving vehicle, the tracks superstructure and the subsoil. As a part of condition monitoring, the outcome of a particular track qualifying process is highly dependent on the type of the running train, the speed of the train, the type of track, the different loads, the properties of primary and secondary suspension, the unsprung masses, the axle distances, the supporting stiffness, the damping and inertial properties of the track system. At any cross-section of the track the location of rail profiles can be fixed in an unloaded state only. Otherwise, the recurrent loading caused by a regular railway traffic can be thought of a time dependent stochastic process which transmits this static setting into a probability distribution.

To eliminate the influence of vehicle parameters, Zobory et al. (1998) have started to construct a specific measuring car equipped with a "measuring wheelset - carrier frame track" subsystem which is discoupled from the underframe dynamically, in order to absolve the carrier frame from the parasitic vibrations of the vehicle which is excited by track unevennesses. The carrier frame was loaded by four nearly constant vertical forces by four congruent air-springs. This configuration is serving as compressed actuators between the carrier frame and the underframe of the measuring car. The layout of the vertical dynamic model of the measuring wheel-set together with the measuring frame as being the major structural elements of this alternative measuring approach is shown in Fig. 3.

The primary goal of establishing this track measuring car was to supply vertical acceleration data for a developed simulation based identification procedure to determine the inhomogeneities in the vertical track stiffness along the longitudinal length direction of the track. Authors used a wavelet-backed approximation to modeling the unknown stiffness variations along the track length. They showed experimentally that this approach corresponds well to track stiffness irregularities that can be observed in railway operation practice (there are some essential peaks at random distance from each other, while the intermediate track sections are disturbed only by a mild narrow bandwidth with low stiffness variations).

This arrangement ensures the transduction of the longitudinal stiffness functions of the track to the vertical acceleration function measured by the measuring frame acceleration sensors mounted over the axlebox as it is seen in Fig. 3.

The longitudinally valid vertically sensed track stiffness parameters are then derived as a minimization of the time integral of the vectorial difference norm square of the measured and the simulated acceleration responses. The estimated optimal values of the vertical stiffness parameters can then be derived from a properly formed least-squares problem, whereas the vertical displacement functions can

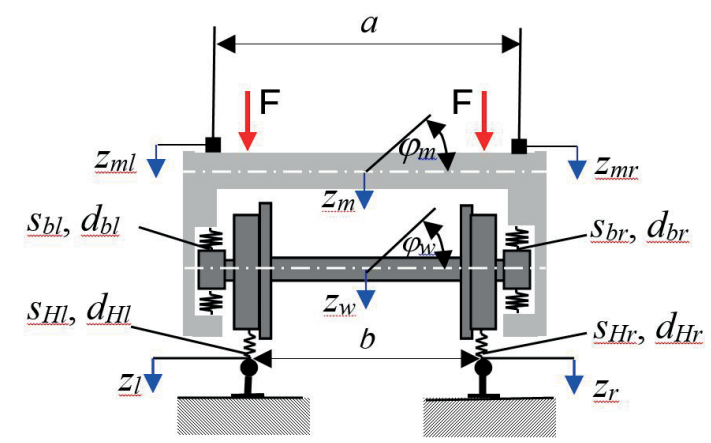

Fig. 3 The vertical dynamical model of the measuring wheel-set and the measuring frame connected with it. (Zobory and Zábori, 2018) 
be determined later on by two successive numerical integrations with respect to time using the acceleration moving average functions (for the sake of comparison).

\subsection{Errors emerging during signal processing}

In the phase of signal processing the accumulating errors from the continuous measurement process give rise to a peculiar issue called a drift. Drift is basically dependent on the movement of the vehicle. In addition, the sources of drift in orientation are not the same as those of in velocity (Fasel, 2017).

In order to calculate orientation, position and velocity, the measured accelerations and angular velocities have to be integrated. Double integration of acceleration to obtain position is unavoidable. Many times occurs in practice that a zero mean acceleration error is assumed, and the double integration with respect to time results in a given position as its mean were zero. Indeed, this mathematical operation that adds to position the integration of a random walk, is not a controllable error and it grows fast. Only by applying a continuous external sensing to bound this type of error can prevent us from this undesired phenomenon (Dissanayake et al., 2001).

Double integration might only be stabilized by adding a high-pass filter. Unfortunately, latter method can only moderately reduce the problem of offset drift (Weston et al., 2015). The different techniques of double integration of the signals extracted from inertial sensors have attracted immense interest in the related literature. We refer to the excellent paper of Naganuma et al. (2008) to find details of this problem.

Lee et al. (2012) proposed a filtering approach including Kalman filter, band-pass filter, compensation filter and phase correction to estimate the track irregularities. Still, for the time being, there is no perfect method for extracting a usable signal from an accelerometer by double integrating it, because their scope is rather limited. Similarly, the noise generated by an accelerometer (and also which comes from an $\mathrm{A} / \mathrm{D}$ converter through signal processing) more or less affects the ultimate results as well (Weston et al., 2015).

In summary, Fig. 4 provides an overview of the steps of the computations about the above discussed processing issues. Observe here the closed-loop feedbacks of the correction process on both of the two branches starting from the measurement data obtained for acceleration and angular velocity.

Gravity is generally greater than the different types of other accelerations hit the vehicle moving along the

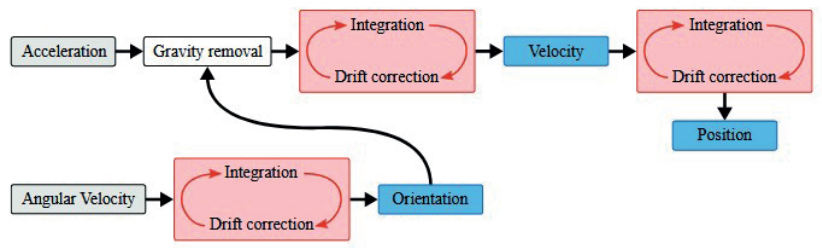

Fig. 4 "Overview of the computation steps required for tracking the sensor's position over time.". (Fasel, 2017:p.22)

track. Eventhough, tiny errors in the attitude may result in large drifts in determining velocity and thus position. The errors in attitude are usually due to false estimation procedures or they are originated in temperature compensation. Measured acceleration is valid referring to the local geographic frame of the inertial sensors, however, it contains gravity. Hence, physical measures have to be given with respect to a global reference frame and gravity must be removed. Even if this correction is done in a proper way, 1-2 degrees of errors in orientation are often remain (Fasel, 2017). Imbalanced gravity removal means that on one axis too much gravity is removed, while a certain amount of gravity is augmented on the other axis. In contrast to orientation drift, the drift in velocity is even greater in magnitude (Fasel, 2017).

To eliminate two types of gyro output errors, the one is a Gaussian noise caused by environmental vibration or electromagnetic radiation, the other can be an installation error when the sensors's sensitive axis is not completely perpendicular to the plane of the train running line, Xing et al. (2015) proposed a mix-filtering approach to obtain a bias free bogie pitch rate, containing time-space domain transformation, double integration, baseline correction and a recursive least squares based adaptive compensation filter.

\subsection{Continuous measurement vs. dedicated measuring coaches}

For a long time past it is well known that a regular track monitoring and track inspection are the main guarantees in achieving high standards for safety and developing an efficient maintenance optimization policy in railway traffic. Measurements of the railroad track could be made by the instrumentation of an in-service vehicle, specifically on a multiple-unit passenger train. A key advantage of this operational manner is that track measurements might be done even in an hour-to-hour basis without interrupting the scheduled operation of railway lines having large traffic, and thus, surveying the track heavily. Gathered data are sent to big database centers where a frequent data processing supports to detect the type and geographic 
position of track faults. Such Track Recording Coaches (TRCs), in order to monitor tracks, are in use today e.g., in Great Britain and belonging to the Network Rail's New Measurement Train (NMT), (Yeo, 2017). The vehicles are capable of measuring all aspects of the track geometry at speeds of up to $140 \mathrm{mph}$. Positioning is done through a combination of GPS, wheel tachometers and by detecting track-mounted electromagnets in the Automatic Warning System (AWS), as a part of the British signaling system.

If an Unattended Geometry Measurement System (UGMS) is used, then additional sensors can be installed, preferably axlebox-mounted accelerometers, to inspect some specific elements of the tracks like switches and crossings, and focusing on to monitor corrugation and joints. This way, the speed of the vehicle is normalizing accelerations and it permits to calculate versines which are mostly independent of the speed of the vehicle (Weston et al., 2015).

If an UGMS is not used, then a bogie-mounted IMU when the primary suspension is stiff - is a perfect solution to monitoring the track, even if the gauge and the twist cannot be sensed. But adding an axlebox accelerometer helps to measure short wavelength data as well. Using such a setting, the vertical acceleration measured on the bogie together with a displacement to the axlebox ensures a first-rate class quality measurement of the vertical rail profile (Weston et al., 2015).

Dedicated measurement vehicles have limitations. They can be expensive to run, requiring specialist crews to operate, as well as being expensive to build initially. Consequently, a railway operating company may only have access to a small number of measurement vehicles. This, coupled with high traffic, causing limited availability on some track, means that measurements may only be possible every one or two months at best in some areas (Yeo, 2017).

\subsection{Accelerometer/Gyroscope characteristics}

An accelerometer measures linear acceleration and gravity. In case of an accelerometer, the magnitude of the signal is biased by gravity and a difficult double integration with respect to time is required to derive displacement plus it has a low signal-to-noise ratio. Contrary to these, in case of a gyroscope, the magnitude of the signal is unbiased, information pertains to bandwidth and frequency available to the extent of zero frequency, a one time integration is sufficient to obtain angular displacement and there is a high signal to noise ratio. Accelerometers must have a range greater than $\pm 100 \mathrm{~m} / \mathrm{s}^{2}$ for bogie, and $\pm 10 \mathrm{~m} / \mathrm{s}^{2}$ for body (Yeo, 2017). Gyros must have a range greater than $\pm 10 \%$ for bogie, and $\pm 1.25 \%$ for body (Yeo, 2017). Very often, railways apply a zero setting during the initial calibration of accelerometers and gyroscopes in order to avoid offset as a harmful behavior at these inertial sensors. Despite these efforts, the offset tends to drift overtime, mainly because of the changes in temperature. Another influencing factors lend themselves in the variations of supply voltage, or other troublesome external impacts, or materials aging.

There is a linear relationship between the acceleration, brought about the static and dynamic effects of the wheel-rail interaction, and the square of the vehicle speed. The acceleration is proportionally related to the amplitude of the irregularities in the track and it is inverse proportionally related to the square of the wavelength. As concerns some particular measurement data, Weston et al. (2015) reported that "A sinusoidal vertical geometrical irregularity with an amplitude of $10 \mathrm{~mm}$ and a wavelength of $50 \mathrm{~m}$ gives an acceleration of $0.32 \mathrm{~m} / \mathrm{s}^{2}$ at $45 \mathrm{~m} / \mathrm{s}$, but gives $0.0032 \mathrm{~m} / \mathrm{s}^{2}$ at $4.5 \mathrm{~m} / \mathrm{s}$." (Weston et al., 2015:p.1027). They found the vertical acceleration measured by an axlebox sensor may even exceed $100 \mathrm{~g}$, especially when a running wheel-pair hits a badly aligned rail joint (Weston et al., 2015).

The observation of long wavelength geometry from an axlebox sensor is almost impossible. We are often facing with this case at low vehicle speeds, e.g. when an in-service vehicle slows down for stopping. Grassie (1996) describes the axlebox-mounted accelerometers as being suitable devices to sensing vertical displacements to conclude from the vertical rail profile. But he mentions also that this measurement is challenging, as there are very strict requirements against the design of such sensors with respect to offset and drift for long wavelengths.

In a spectacular contrast to the previous considerations, placing an accelerometer sensor on the bottom of the body of the vehicle bears much less difficulty, because here, the typical acceleration values become less than $1 \mathrm{~g}$. Hence, the long wavelength geometry induced by small accelerations are more simple to measure on the body. On the bogie, the vertical accelerations are usually only around or less than $10 \mathrm{~g}$. Furthermore, whatever the choice is for the placement of the acceleration sensors, the values of the measurements are speed dependent. It can be said that the faster is the movement of the train, the greater the acceleration values are likely to occur at the same section of the track.

Regarding the use of the gyroscope sensors, several authors expressed that they perform very effectively on 
the bogie, but they are much less beneficial on the axlebox (Weston et al., 2015). Gyros measuring yaw and roll rates have been employed successfully for long ago (Lewis and Richards, 1988), but other authors claimed in favor of using bogie-mounted pitch rate gyros on the in-service vehicles (Weston et al., 2015). Gyroscopes can provide valuable measurement data at low vehicle speeds compared to the conventionally used accelerometers, particularly in the case of frequent station stops at the regular in-service train sets (Weston et al., 2007; Yeo, 2017).

\section{Kinematical background of inertial navigation and measurement systems}

In this section, we present a concise description about the physical background of the inertial navigation problem. In this framework, the required measurements are made by accelerometers and gyroscopes which devices are installed on a dedicated or in-service vehicle. The sensed transitional and rotational motions are used to obtain the position of the vehicle. The totality of this data set comprises both of these two types of measurements and enables the users to determine the true motion of the vehicle within a properly chosen inertial frame of reference, and thus, to calculate its position. Our brief discussion about the essential functions that an inertial navigation system must perform will follow the excellent book of Titterton and Weston (1997). Firstly, it is necessary to define an adequate reference frame for any inertial navigation and measurement system. Each of these frames represents an orthogonal axis set. This triaxial coordinate system is interpreted as a right-handed one.

Consider a fixed environment without acceleration and rotation. To estimate the constituent members of the acceleration with respect to a space-fixed reference system, the measured elements of the specific force and the gravitational estimates should be summed. Let $\boldsymbol{r}$ denote the column vector (position vector) of an arbitrarily chosen point $\mathrm{P}$ tied to origin $\mathrm{O}$ of the reference frame. Now, let the acceleration of $\mathrm{P}$ be defined in the $\mathrm{i}$-frame (inertial frame with its origin at the centre of the Earth and axes which are non-rotating) and denoted by the index $i$, as described by:

$\boldsymbol{a}_{\mathrm{i}}=\left.\frac{\mathrm{d}^{2} \boldsymbol{r}}{\mathrm{d} t^{2}}\right|_{\mathrm{i}}=\boldsymbol{f}^{\mathrm{i}}+\boldsymbol{g}^{\mathrm{i}}=\boldsymbol{C}_{\mathrm{b}}^{\mathrm{i}} \boldsymbol{f}^{\mathrm{b}}+\boldsymbol{g}^{\mathrm{i}}$,

where triaxial accelerometers will provide us the measure of the specific force, $\boldsymbol{f}$ that acts at point P. In Eq. (1), $g$ represents the so called mass attraction gravitation vector. The specific force is usually given in a b-frame (in a body fixed axis set, denoted by $\boldsymbol{f}^{\mathrm{b}}$ which is an orthogonal coordinate system aligned with the roll, pitch and yaw axes of the vehicle within the established navigation system). The specific force should be pre-multiplied by the direction cosine matrix, $\boldsymbol{C}_{\mathrm{b}}^{\mathrm{i}}$. With proper numerical integration of the navigation equation represented by Eq. (1), the velocity and position of the vehicle can be derived. The velocity of point $\mathrm{P}$ with respect to the to the $\mathrm{i}$-frame is produced by the first integral and given as

$\boldsymbol{v}_{\mathbf{i}}=\left.\frac{\mathrm{d} \boldsymbol{r}}{\mathrm{d} t}\right|_{\mathrm{i}}$,

whilst a suitable next integration yields the position of point $P$ in the same reference frame. The $3 \times 3$ sized direction cosine matrix, $\boldsymbol{C}_{\mathrm{b}}^{\mathrm{i}}$, whose columns are unit vectors in body frame which were then projected along the reference axes and may be calculated from the gyroscopes' measurements for the angular rates, is seen in Eq. (3):

$$
\dot{\boldsymbol{C}}_{\mathrm{b}}^{\mathrm{i}}=\boldsymbol{C}_{\mathrm{b}}^{\mathrm{i}} \Omega_{\mathrm{ib}}^{\mathrm{b}}=\boldsymbol{C}_{\mathrm{b}}^{\mathrm{i}}\left[\begin{array}{rrr}
0 & -r & q \\
r & 0 & -p \\
-q & p & 0
\end{array}\right],
$$

where the second factor, $\Omega_{\mathrm{ib}}^{\mathrm{b}}$ of the matrix product is composed of the elements of the vector, $\omega_{\mathrm{ib}}^{\mathrm{b}}=[p, q, r]^{\top}$ representing the turn rate of the body with respect to the i-frame, as directly measured by the gyros.

In this system, it is required to calculate and use the speed of the vehicle with respect to the Earth, called ground speed in the i-frame axes, which is denoted by the symbol $\boldsymbol{v}_{\mathrm{e}}^{\mathrm{i}}$ (Titterton and Weston, 1997):

$$
\left.\frac{\mathrm{d} \boldsymbol{v}_{\mathrm{e}}}{\mathrm{d} t}\right|_{\mathrm{i}}=\dot{\boldsymbol{v}}_{\mathrm{e}}^{i}=\boldsymbol{C}_{\mathrm{b}}^{\mathrm{i}} \boldsymbol{f}^{\mathrm{b}}-\omega_{\mathrm{ie}}^{\mathrm{i}} \times \boldsymbol{v}_{\mathrm{ie}}^{\mathrm{i}}-\omega_{i e}^{i} \times\left[\omega_{\mathrm{ie}}^{\mathrm{i}} \times \boldsymbol{r}\right]+\boldsymbol{g}^{\mathrm{i}} .
$$

In Eq. (4), the first member, $\boldsymbol{C}_{\mathrm{b}}^{\mathrm{i}} \boldsymbol{f}^{\mathrm{b}}$, represents the specific force acceleration; whilst the second one in this expression is the Coriolis acceleration given in the form of a vector product induced by its velocity over the surface of the rotating Earth; finally, the third member in Eq. (4) describes the centripetal acceleration impacting due to the rotating Earth which cannot make to be separately distinguishable from the gravitational acceleration which arises through mass attraction, $g$. Due to their small effects in the b-frame, the latter two accelerations are usually neglected from Eq. (4) (Titterton and Weston, 1997).

Gyroscopic sensors are used to instrument a reference co-ordinate frame within a vehicle which is free to rotate about any direction. The calculated attitude of the vehicle may be stored as a set of numbers in a computer within 
the vehicle. The stored attitude is updated as the vehicle rotates using the measurements of the turn rates provided by the gyroscopes (Titterton and Weston, 1997). Positive rotations about each axis are interpreted as spinnings into clockwise directions when looking along the axis from the origin. A comprehensive block diagram representation of the inertial frame mechanization system is exhibited in Fig. 5 (Titterton and Weston, 1997:p.29).

The rate of change of the direction cosine matrix, $\boldsymbol{C}_{\mathrm{b}}^{\mathrm{n}}$ with time given in an $\mathrm{n}$-frame (the navigation frame is a local geographic frame which has its origin at the location of the navigation system, i.e. point $\mathrm{P}$, and axes aligned with the directions of north, east and the local vertical down) yields, in a limiting sense, as $\Delta t \rightarrow 0$, and $\psi, \phi$ and $\theta$ are the (small) Euler rotation angles through which the b-frame has rotated over the time interval $\Delta t$ about its yaw, pitch and roll axes (see in Fig. 6), respectively:

$\dot{\boldsymbol{C}}_{\mathrm{b}}^{\mathrm{n}}=\boldsymbol{C}_{\mathrm{b}}^{\mathrm{n}} \Omega_{\mathrm{nb}}^{\mathrm{b}}=\boldsymbol{C}_{\mathrm{b}}^{\mathrm{n}}\left[\begin{array}{rrr}0 & -\omega_{z} & \omega_{y} \\ \omega_{z} & 0 & -\omega_{x} \\ -\omega_{y} & \omega_{x} & 0\end{array}\right]$.

Each rotation in Eq. (5) is then separated into three distinct direction cosine matrices, entries of which are composed of appropriate sine and cosine functions of the Euler angles. For more details see Titterton and Weston (1997). Due to the very small angle rotations here, these sines and cosines become zero and one, respectively, in the skew symmetric matrix given in Eq. (5). Hence, in terms of Euler rotations, the following approximate direction cosine matrix may be obtained, which relates from the body to the reference axes:

$\boldsymbol{C}_{\mathrm{b}}^{\mathrm{n}} \cong\left[\begin{array}{rrr}1 & -\psi & \theta \\ \psi & 1 & -\phi \\ -\theta & \phi & 1\end{array}\right]$.

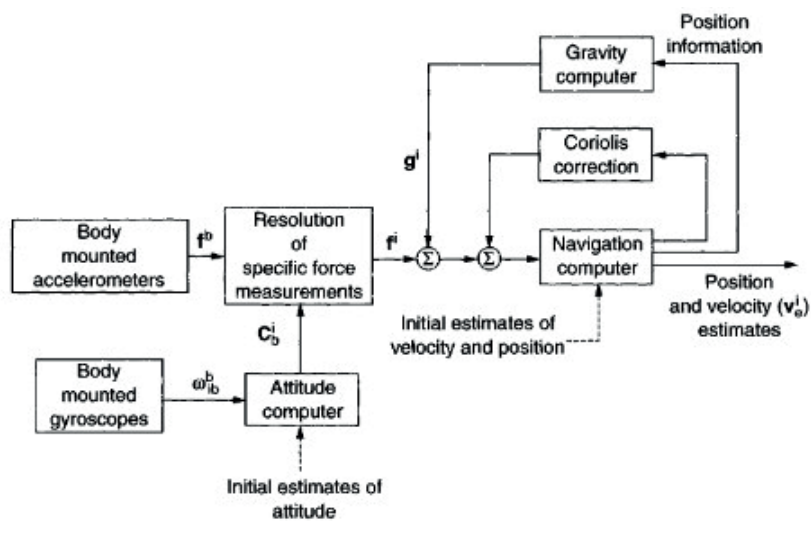

Fig. 5 Block diagram representation of an inertial navigation system (Titterton and Weston, 1997:p.29)
According to the gimbal suspension analogy (a pivoted support that allows the rotation of an object about a single axis), $\psi, \phi$ and $\theta$ are the gimbal angles and, $\dot{\psi}, \dot{\phi}$ and $\dot{\theta}$ are the gimbal rates which are directly related to the body rates, $\omega_{x}, \omega_{y}$ and $\omega_{z}$.

\section{Notions and description of train motions}

A rail-road track is usually represented by a single parameter, $s$ associated with the change of the general position coordinate of the moving train set over the time interval $\Delta t$. The well-known kinematic motion equation can be expressed as:

$s=s_{0}+\dot{s} t+\frac{1}{2} \ddot{s} t^{2}+\ldots$,

producing the displacement (distance) into the longitudinal direction on the effects of the velocity of the train which is the first time derivative of $s$ and the train acceleration or deceleration (as a result of traction or brakes) as the second time derivative of the general parameter $s$.

To study the motion of a train on the track properly, the angular coordinates should be introduced. The deviation of the track position from geographic north is called heading and denoted by $\psi(s)$. A change in heading is termed as a curvature of the track, $\mathrm{d} \psi(s) / \mathrm{d} s$, which can be expressed by the reciprocal of the radius for a given segment of the track (Heirich et al., 2011). Moving in a curvature results in lateral acceleration as a response to the front wheel pair input. Irregularities, on the effect of centrifugal forces may have a large impact on the riding quality of the performance of the running vehicle, if the velocity is high $(v>250 \mathrm{~km} / \mathrm{h})$ and the radius is relatively small ( $R<5,000 \mathrm{~m})$, (Yi, 2018:p.311).

Turning in a circle requires a vehicle to have a centripetal acceleration inwards on the turn. To avoid serious physical balancing problems, the track is banked inwards, i.e. the inner rail is lower than the outer rail (superelevation/cant). The bank angle, $\theta(s)$ is the lateral inclination of the track. The change in the bank, $\mathrm{d} \theta(s) / \mathrm{d} s$ is called a bank rate. The horizontal inclination, simply saying a slope, $\phi(s)$ in the longitudinal direction of the track can be ascending or descending. The change in the altitude level, $\mathrm{d} \phi(s) / \mathrm{d} s$ is termed a pitch rate. For more details about these geometric interpretations, see e.g. Heirich et al. (2011).

Railway track routes are designed as a combination of their basic elements: straight lines, circles and transition curves (clothoids, S-shaped curves, etc.). Geometric design of the transition segments attempts to achieve a linear increase of curvature (Heirich et al., 2011). 
There have some specific elements of the railway tracks. They are the switches, crossings, rail joints, etc. which cause strong lateral and vertical jerk. In addition, they may result in a sudden growth in the turn rate and both in the lateral and the vertical accelerations. It is interesting to note here that there are over 300,000 of such units within the networks of the EU 28 countries (Capacity 4 Rail, 2015).

Using a three dimensional coordinate system (b-frame) for the train (or for a structural element of it, i.e. the bogie), the different rotational motions around the corresponding translational axes are expressed by the angular velocities and can be measured by gyroscopic sensors, denoted by $\omega_{\text {yaw }}, \omega_{\text {pitch }}$ and $\omega_{\text {roll }}$. An other coordinate system, the n-frame stands for measuring the attitude of the train frame by rotation angles between the axes with reference to gravity and geographical north of the Earth. This reference frame has a horizontal plane which is perpendicular to gravity. Rotation angles to the train frame are: $\psi$ for the heading of the train (rotate through angle $\psi$ about reference $z$-axis), $\phi$ for slope angle (rotate through angle $\phi$ about a new $y$-axis) and $\theta$ for bank angle (rotate through angle $\theta$ about a new $x$-axis), as they are displayed in Fig. 6 .

A rotation around an axis is taken to be positive, if it happens in clockwise direction while looking along the axis from the origin, as it is indicated in Fig. 6. Observe also that the $z$-axis is pointing downwards. It follows that $\psi=\phi=\theta=0$ means that the vehicle is positioned to the north direction and is standing perpendicular to gravity. It should be noted that one should keep the order of the rotations.

The movements experienced by an IMU have to be interpreted in three dimensions. As of in the six degreeof-freedom model depicted in Fig. 6, the bogie may move in 3 perpendicular axes and may rotate about those axes.

The geometry frame is oriented with the bogie of the IMU so that axis $x$ leads always in parallel to the

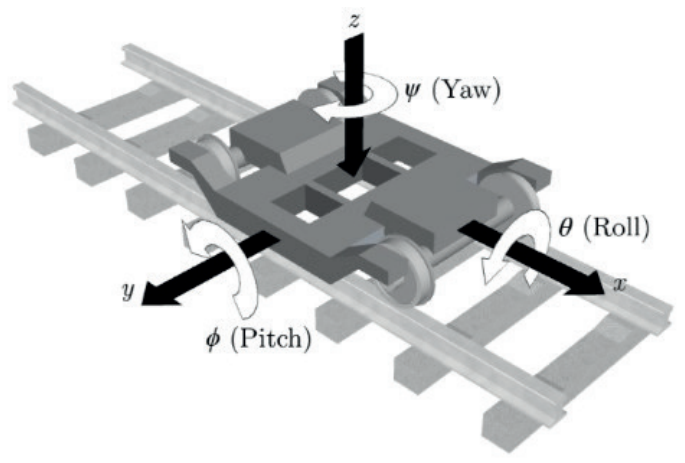

Fig. 6 Interpretation of a six degree-of-freedom model applied to a vehicle bogie (Yeo, 2017:p.52) track. The IMU is mounted on the bogie such that each of its faces are always parallel to one of the three axes. Therefore, each of the three accelerometers directly measures the accelerations pointing into the predefined axes, $x, y$ and $z$, and each of the three gyroscopes directly measure the changes in the rotation angles, $\psi, \phi$ and $\theta$. The measured accelerations will vary depending on the actual placement of the IMU on the bogie. The same is not true for the angular velocities, which will remain invariant in each axis when measured at different locations on the bogie by the gyros (Yeo, 2017).

\section{Conclusions}

In this overview, the most recent findings in condition monitoring of railway track geometry have been collected and reviewed based on a comprehensive literature research. The kinematics of inertial navigational systems and basic train motions on the effects of the different geometric and geographic train positions have also been summarized. In conclusion, this survey has shown that there is not any universally applicable configuration of a railway track condition monitoring system which would incorporate the required measurements of track defects of every kind. A choice for the actual instrumentation of such a system in case of a given railway network is a matter of engineering and economic rationale.

Future research should address more focus on condition monitoring issues of high speed railway lines like the SNCF TGV Iris 320 dedicated track recording train, which is equipped by pantograph, laser diodes and transversal linear, and longitudinal matrix cameras to offer simultaneous two axis recording.

Which clearly observable at leading railway companies means that a really effective track geometry measurement system should be composed of a six degree-of-freedom inertial measurement system (IMU) including closed loop configured accelerometers with possibly high performance operating parameters that are installed on the bogie, plus, sometimes, it would be expedient to mount displacement transducers between the bogie and the axle boxes. Eventually, an optical attitude determination system might also be added. Most European and Far-Eastern railway companies strive to employ in-service vehicles. The obtained signals should be high-pass filtered and have a baseline and phase correction to eliminate the errors emerging in the double integration process to derive more accurate orientation, velocity, and position information. 


\section{References}

Ackroyd, P., Angelo, S., Nejikovsky, B., Stevens, J. (2002) "Remote Ride Quality Monitoring of Acela Train Set Performance", In: 2002 ASME/IEEE Joint Rail Conference, Washington, DC, USA, pp. $171-178$.

https://doi.org/10.1115/RTD2002-1650

Capacity 4 Rail (2015) "Operational failure modes of switches and crossings", Collaborative Project, UIC Voestalpine VAE GmbH, VAE. [online] Available at: www.capacity4rail.eu [Accessed: 21 October 2018]

Charles, G., Goodal, R., Dixon, R. (2008) "Model-based condition monitoring at the wheel-rail interface", Vehicle System Dynamics, 46(1), pp. 415-430. https://doi.org/10.1080/00423110801979259

CH Robotics (2012) "Estimating Velocity and Position Using Accelerometers", Research document 1.0, Australia, AN-1007.

Destek, M. (1974) "A vasúti pálya és a jármű kölcsönhatásának rendszerszerü vizsgálata" (Investigation of the interaction of track/vehicle system in approach of system theory), Közlekedéstudományi Szemle, 24(6), pp. 271-276. (in Hungarian)

Dissanayake, G., Sukkarieh, S., Nebot, E., Durrant-Whyte, H. (2001) "The aiding of a low-cost strapdown inertial measurement unit using vehicle model constraints for land vehicle applications", IEEE Transactions on Robotics and Automation, 17(5), pp. 731-747. https://doi.org/10.1109/70.964672

Fasel, B. (2017) "Drift Reduction for Inertial Sensor Based Orientation and Position Estimation", PhD Thesis, École Polytechnique Fédérale de Lausanne, Switzerland.

Grassie, S. L. (1996) "Measurement of railhead longitudinal profiles: a comparison of different techniques", Wear, 191(1-2), pp. 245-251. https://doi.org/10.1016/0043-1648(95)06732-9

Heirich, O., Lehner, A., Robertson, P., Strang T. (2011) "Measurement and analysis of train motion and railway track characteristics with inertial sensors", In: 2011 14th International IEEE Conference on Intelligent Transportation Systems (ITSC), Washington, DC, USA, pp. 1995-2000. https://doi.org/10.1109/ITSC.2011.6082908

Jochim, H., Lademann, F. (2009) "Planung von Bahnanlagen" (Planning of Railway Facilities), Carl Hanser Verlag, München, Germany. (in German)

King, S. (2004) "The UK's fastest track monitoring system as used on the channel tunnel rail link", In: IEE Seminar. Railway Condition Monitoring - Why? What? How?, Derby, UK, pp. 11-32. https://doi.org/10.1049/ic:20040163

Lee, J. S., Choi, S., Kim, S. S., Park, C., Kim, Y. G. (2012) "A Mixed Filtering Approach for Track Condition Monitoring Using Accelerometers on the Axle Box and Bogie", IEEE Transactions on Instrumentation and Measurement, 61(3), pp. 749-758. https://doi.org/10.1109/TIM.2011.2170377

Lewis, R. B., Richards, A. N. (1988) "A compensated accelerometer for measurement of railway track crosslevel", IEEE Transactions on Vehicular Technology, 37(3), pp. 174-178.

https://doi.org/10.1109/25.16544
Mirabadi, A., Schmid, F., Mort, N. (2003) "Multisensor Integration Methods in the Development of a Fault-Tolerant Train Navigation System", The Journal of Navigation, 56(3), pp. 385-398. https://doi.org/10.1017/S0373463303002364

Molodova, M., Li, Z. Dollevoet, R. (2011) "Axle box acceleration: Measurement and simulation for detection of short track defects", Wear, 271(1-2), pp. 349-356. https://doi.org/10.1016/j.wear.2010.10.003

Naganuma, Y., Kobayashi, M., Nakagawa, M., Okumura, T. (2008) "Condition monitoring of Shinkansen tracks using commercial trains", In: 4th IET International Conference on Railway Condition Monitoring (RCM 2008), Derby, UK, Article ID 14. https://doi.org/10.1049/ic:20080321

Nielsen, J., Berggren, E., Lölgen, T., Müller, R., Stallaert, B., Pesqueux, L. (2013) "Overview of Methods for Measurement of Track Irregularities Important for Ground-Borne Vibration", UIC Collaborative Project Report, Rivas, Nicaragua, SCP0-GA-2010-2657.

Roberts, C., Weston, P., Ling, C. S., Goodman, C. (2004) "New methods for track monitoring", In: IEE Seminar Railway Condition Monitoring - Why? What? How?, Derby, UK, pp. 64-78.

Szabó, A., Zobory, I. (1998) "On deterministic and stochastic simulation of wheel and rail profile wear process", Periodica Polytechnica Transportation Engineering, 26(1-2), pp. 3-17.

Titterton, D. H., Weston, J. L. (1997) "Strapdown Inertial Navigation Technology", Peregrinus, Stevenage, UK.

Trehag, J., Handel, P., Ogren, M. (2010) "Onboard Estimation and Classification of a Railroad Curvature", IEEE Transactions on Instrumentation and Measurement, 59(3), pp. 653-660. https://doi.org/10.1109/TIM.2009.2025082

Ward, C. P., Weston, P. F., Stewart, E. J. C., Li, H., Goodall, R. M., Roberts, C., Mei, T. X., Charles, G., Dixon, R. (2011) "Condition Monitoring Opportunities Using Vehicle-Based Sensors", Proceedings of the Institution of Mechanical Engineers, Part F: Journal of Rail and Rapid Transit, 225(2), pp. 202-218. https://doi.org/10.1177/09544097JRRT406

Weston, P. F., Ling, C. S., Roberts, C., Goodman, C. J., Li, P., Goodall, R. M. (2007) "Monitoring vertical track irregularity from in-service railway vehicles", Proceedings of the Institution of Mechanical Engineers, Part F, Journal of Rail and Rapid Transit, 221(1), pp. $75-88$. https://doi.org/10.1243/0954409JRRT65

Weston, P. F., Roberts, C., Yeo, G., Stewart, E. (2015) "Perspectives on railway track geometry condition monitoring from in-service railway vehicles", Vehicle System Dynamics: International Journal of Vehicle Mechanics and Mobility, 53(7), pp. 1063-1091. http://doi.org/10.1080/00423114.2015.1034730

Xing, Z., Chen, Y., Qing, Y. (2015) "On-line monitoring of vertical long wavelength track irregularities using bogie pitch rate", Journal of Vibroengineering, 17(1), pp. 216-228.

Yazawa, E., Takeshita, K. (2002) "Development of Measurement Device of Track Irregularity using Inertial Mid-chord Offset Method", Quarterly Report of RTRI, 43(3), pp. 125-130. https://doi.org/10.2219/rtriqr.43.125 
Yeo, G. J. (2017) "Monitoring railway track condition using inertial sensors on an in-service vehicle", PhD Thesis, University of Birmingham, UK.

Yi, S. (2018) "Dynamic Analysis of High-Speed Railway Alignment. Theory and Practice", Academic Press, London, UK.

ZG Optique SA (2016) "Track Geometry Measuring System", Company Report, St-Aubin, Switzerland.

Zobory, I. (2015) "A vasúti pálya-jármü rendszer dinamikája-mérése-minősítése" (Dynamics-Measurement-Qualification of railway track-vehicle systems), Közlekedéstudományi Szemle, 65(1), pp. 6-19. (in Hungarian)

Zobory, I., Békefi, E., Zábori, Z. (1998) "Simulation backed identification of vertical track stiffness functions by using wavelets", In: Proceedings of $6^{\text {th }}$ Mini Conference on Vehicle System Dynamics, Identification and Anomalies, Budapest, Hungary, pp. 151-159.
Zobory, I., Péter, T. (1987) "Dynamic processes caused by track unevennesses in braked railway vehicles", Periodica Polytechnica Transportation Engineering, 15(2), pp. 171-183.

Zobory, I., Zábori, Z. (1996) "Track qualification method and its realization based on system dynamics", In: Proceedings of $4^{\text {th }}$ Mini Conference on Vehicle System Dynamics, Identification and Anomalies, Budapest, Hungary, pp. 173-181.

Zobory, I., Zábori, Z. (2018) "Track qualification method using system dynamics based parameter identification procedure", In: Proceedings of $16^{\text {th }}$ Mini Conference on Vehicle System Dynamics, Identification and Anomalies, Budapest, Hungary. (to appear) 